\title{
Diverse mechanisms for epigenetic imprinting in mammals
}

\author{
Daniel Andergassen ${ }^{1,2 *}$, Zachary D. Smith ${ }^{1,3,4 *}$, John L. Rinn ${ }^{5}$ and, Alexander \\ Meissner ${ }^{1,3,6,7}$ \\ 1Department of Stem Cell and Regenerative Biology, Harvard University, Cambridge, MA, USA
${ }^{2}$ Institute of Pharmacology and Toxicology, Technical University Munich (TUM), 80802, Munich, Germany
${ }^{3}$ Broad Institute of MIT and Harvard, Cambridge, MA, USA
${ }^{4}$ Yale Stem Cell Center, Department of Genetics, Yale School of Medicine, New Haven, CT, USA
${ }^{5}$ Department of Biochemistry, University of Colorado Boulder, Boulder, United States
${ }^{6}$ Department of Genome Regulation, Max Planck Institute for Molecular Genetics, Berlin Germany
${ }^{7}$ Institute of Chemistry and Biochemistry, Freie Universität Berlin, Berlin, Germany
}

Correspondence: meissner@molgen.mpg.de

\section{SUMMARY}

Genomic imprinting and $\mathrm{X}$ chromosome inactivation $(\mathrm{XCl})$ require epigenetic mechanisms to direct allele-specific expression. Despite their critical roles in embryonic development, how universal epigenetic regulators coordinate these specific tasks from single locus to chromosome-scale remains understudied. Here, we systematically disrupted multiple essential epigenetic pathways within polymorphic F1 zygotes to examine postimplantation effects on canonical and non-canonical genomic imprinting as well as $X$ chromosome inactivation. We find that DNA methylation and Polycomb group repressors are both indispensable for autosomal imprinting, albeit at distinct gene sets. Moreover, the extraembryonic ectoderm relies on a broader spectrum of unique imprinting mechanisms, including non-canonical targeting of maternal endogenous retrovirus (ERV) driven promoters by G9a. We further utilize our data to identify Polycomb dependent and independent gene clusters on the imprinted $X$ chromosome, which appears to reflect distinct domains of Xistmediated suppression. Our data has allowed us to assemble a comprehensive inventory of the epigenetic mechanisms utilized in eutherian mammals to maintain parent-specific imprinting, including an expanded view of the placental lineage that comprises multiple unique pathways.

\section{INTRODUCTION}

Mammals have two autosomal gene copies, one inherited from each parent. The vast majority of genes are biallelically expressed, while a small subset is expressed in a parent of origin-specific fashion. Genomic imprinting is maintained via distinct epigenetic mechanisms to propagate information from the oocyte or sperm into the next generation, and can regulate the expression of nearby genes in cis as a secondary mechanism. Multiple loci have been thoroughly dissected to understand how this process is carried out molecularly. For example, epigenetic modifications can repress long non-coding RNAs (IncRNAs) that otherwise target and repress nearby genes. For decades, DNA methylation was considered the only epigenetic modification that could be transmitted from the germline into the subsequent progeny. Recently, oocyte-specific trimethylation of H3 on lysine 27 (H3K27me3) was 
found to transiently imprint several loci within preimplantation embryos, of which some transition to a more permanent DNA methylated state to preserve paternal expression (Chen et al., 2019; Hanna et al., 2019; Inoue et al., 2017a). Noncanonical imprinting by $\mathrm{H} 3 \mathrm{~K} 27 \mathrm{me} 3$ appears to primarily function in placental development and regulates genes with critical functions in this lineage. This surprising alternative imprinting strategy implies that mammals may use other mechanisms beyond DNA methylation to instruct parent-specific regulation.

Notably, oocyte-specific H3K27me3 also serves as a maternal imprint for the IncRNA Xist, which triggers paternal $\mathrm{X}$ chromosome inactivation $(\mathrm{XCl})$ in mouse preimplantation embryos and extraembryonic tissues (Inoue et al., 2017b). While similar to non-canonical $\mathrm{H} 3 \mathrm{~K} 27$ me3-based regulation of single loci, $X$ inactivation is capable of relaying this information to suppress an entire chromosome and also operates through several classic epigenetic suppression pathways (Żylicz and Heard, 2020). Failure to establish or maintain either $\mathrm{XCl}$ or genomic imprinting results in embryonic lethality, emphasizing the developmental importance of these interrelated processes. However, the molecular relationship between imprinted $\mathrm{XCl}$ and other H3K27me3 regulated loci, or what distinguishes these strategies from canonical, DNA methylation-based imprinting, remains unresolved.

To compare and contrast the developmental roles of epigenetic regulators in these processes, we used reciprocal crosses between highly polymorphic strains to investigate allele-specific expression in E6.5 epiblast (the embryonic progenitor) and extraembryonic ectoderm (ExE, the placental progenitor, which exhibits imprinted $\mathrm{XCl}$ ). With these data, we generate a comprehensive map of all the imprinted genes in both embryonic and extraembryonic lineages, as well as genes that escape imprinting on the inactive $X$ chromosome. Then, we systematically perturbed multiple epigenetic pathways, including DNA methylation, Polycomb-based repression, and Histone 3 Lysine 9 methylation (H3K9me), to understand their primary contributions to parent-specific expression. We find that DNA methylation appears to function primarily at previously described canonical Imprint Control Regions (ICRs) within both lineages, whereas the early placenta exhibits a greater diversity of imprinting mechanisms. For example, we identify an H3K9 methylation-based mechanism that recognizes and suppresses ERV-driven promoters exclusively at maternal loci. Furthermore, our observations pinpoint the explicit dependencies of distinct $X$ chromosomal regions on the Polycomb repressor complexes (PRCs), including gene clusters that appear to rely on Xist recruitment alone. We also find that PRC2 has independent roles in Xist imprinting and chromosome-wide silencing. Finally, our data set enables us to generate a complete inventory of parent-specific expression signatures as they depend on epigenetic pathways.

\section{RESULTS}

\section{Capturing allele-specific expression in the early embryo and placenta}

To explore parent-specific expression within the early embryonic and extraembryonic lineages, we conducted reciprocal crosses between CAST/EiJ (CAST) and B6D2F1/J (BDF1) strain animals, isolated embryonic day (E)6.5-stage epiblast (Epiblast) and extraembryonic ectoderm (ExE), and performed RNA-seq (Figure 1A, Table S1 
sheet A). On average, this approach allowed us to call allele-specific expression for 15,199 and 13,729 genes for the Epiblast and ExE, respectively, varying between 14,136-20,567 and 13,500-14,143 genes per replicate (informative gene $\geq 10$ reads SNPs overlap, see Methods). Unsupervised clustering of gene expression confirmed epiblast and ExE lineage identity and sample purity. Overall, allelic ratios exhibited expected patterns: biallelic expression for autosomal genes, skewed $\mathrm{XCl}$ that favors expression of the CAST allele in Epiblast (Calaway et al., 2013), and imprinted XCI in ExE (Figure S1A-B). For autosomal imprinting, we identified 22 Epiblast- and 41 ExE-specific imprinted genes, with 19 shared between lineages (Figure 1B-D, Table S1 sheet B-C). We also detected the seven known non-canonical imprinting genes (Gab1, Sfmbt2, Slc38a4, Phf17, Smoc1, Platr20, and Gm32885) in ExE, as well as seven putative, novel imprinted genes (Epiblast $n=1$ and ExE $n=6$ ). Our newly discovered imprinted loci are located in proximity to known imprinted regions and include six imprinted IncRNAs as well as maternally dominant expression of Brachyury $(T)$ and Pnldc1.

To explore chromosome-level regulation, we next examined expression of $\mathrm{X}$ linked genes in female ExE samples, which undergo imprinted $\mathrm{XCl}$ (Figure 1E, Table S1 sheet D). Of 335 informative X-linked genes, $290(86.5 \%)$ are maternally expressed, 11 (3.3\%) escape inactivation ('escaper,' excluding Xist), and 33 (9.85\%) escape $X$ inactivation in a strain-specific manner (Cast $n=15$, and BDF1 $n=18$ ). Half of escaper genes cluster in proximity to the $1.85-\mathrm{Mb} X$ inactivation center candidate region defined in Chadwick et al. (Ref (Chadwick et al., 2006)), while others include notable epigenetic regulators (e.g., Suv38h1, Kdm6a, Kdm5c, Figure 1F). In summary, we mapped the parental-specific transcriptional landscape in the early embryonic and extraembryonic lineage for both males and females, allowing us to examine autosomal and X-chromosome-specific imprinting.

\section{Determining imprinting regulators by zygotic genome perturbation}

Our comprehensive map of parent-specific expression allows us to systematically investigate the roles of key epigenetic pathways by Cas9-based genetic disruption in zygotes (Grosswendt et al., 2020; Smith et al., 2017) (Figure 2A, see Methods). For this study, we disrupted the DNA methyltransferase Dnmt1, the histone 3 lysine 9 (H3K9) methyltransferases G9a and GLP (target genes Ehmt1 and Ehmt2, double $\mathrm{KO}$ ), as well as the PRC1 and PRC2 complexes individually (target genes: Rnf2 and Eed, respectively) in BDF1xCAST zygotes. Epiblast and ExE samples were collected from 30 knockout embryos at E6.5 and processed for bulk RNA sequencing (Table S1 sheet E-F). We validated gene disruption by comparing the expression profile and level of the target gene between wildtype and KO embryos (Figure S2A-B) and excluded imprinted genes with less than two informative allelic ratios in any regulator disruption, resulting in 19 epiblasts and 37 ExE imprinted genes. Next, we performed unsupervised clustering of autosomal expression levels and confirmed all samples preserved their overall lineage identity (Figure S2C). Within the Epiblast and ExE clusters, we found that $\triangle D n m t 1$ and $\triangle G 9 a-G L P$ embryos co-cluster, as do $\triangle R n f 2$ and $\Delta E e d$, in keeping with their more similar functions (Auclair et al., 2016; Jiang et al., 2020). 
The extraembryonic lineage uses an expanded set of imprinting mechanisms

To test which epigenetic regulators are responsible for parent-specific regulation, we first examined the allelic landscape of autosomes. We observed biallelic expression of imprinted silencing for most identified alleles within the $\Delta D n m t 1$ Epiblast, the majority of which are also deregulated in ExE (Figure 2B, Table S1 sheet G). In contrast, the ExE includes several additional dependencies that largely reflect parent of origin and epigenetic mechanism. For example, G9a appears to regulate a discrete set of paternally imprinted genes, which also depend to a lesser degree on PRC regulators. In contrast, Rnf2 and Eed mutants preferentially influence the expression of maternal imprints and do so independently of either H3K9 or DNA methylation (Figure 2C, Table S1 sheet $\mathbf{H}$ ). After examining our mutant data, only five imprinted genes (three in Epiblast and two in ExE) cannot be explained by at least one of these key pathways and remain imprinted across our mutant cohorts (delta allelic ratio $<20 \%$ ).

We also find that the PRCs stabilize imprinted $X$ inactivation, with PRC2 disruption more severe than PRC1 (Figure 2D, Figure S2D). Loss of PRC2 regulation during $\mathrm{XCl}$ mirrors the response of $\mathrm{PRC2}$-dependent imprints, with muted reactivation of the silenced allele or paternal chromosome, respectively. Notably, we do not observe disrupted Xist imprinting or changes in allelic expression for $\sim 36 \%$ of $X$-linked genes across all mutant regulators examined. Together, the preserved imprinting of Xist and the muted reactivation of paternal alleles suggest that PRC2 acts to stabilize subsets of Xist IncRNA target genes, an observation we explore in greater detail below.

\section{G9a-GLP control a discrete set of non-canonical imprinted genes}

In Epiblast, the majority of imprinted genes appear to depend primarily on DNA methylation ( $n=14$ out of 19 genes, 3 of which do not change in any KO, Figure 3A left, Figure S3A). Zdbf2 and S/c38a4 are notable exceptions: Zdbf2 is reactivated in both Rnf2 and Eed mutants, whereas S/c38a4 primarily relies on G9a-GLP, in line with previous reports (Auclair et al., 2016; Greenberg et al., 2017). Notably, most epiblast-associated imprinted regions exhibit similar changes and dependencies in the ExE, suggesting that these are constitutive and do not depend on their respective lineage (Figure 3A right, Figure 3B, Table S1 sheet I). In contrast, 20 out of 37 total imprinted genes in the EXE are DNA methylation independent (Figure 3A right). Of these, seven maternally expressed loci are chromatin-dependent and predominantly rely on PRC1 and 2 (Figure 3B top). All of these are either known targets or in local proximity to paternally expressed IncRNAs Kcnq1ot1 (SIc22a18, Alsc/2, Tssc4, R74862, Cd81) and Airn (T,Phlda2), which are believed to recruit these complexes to enable silencing of paternal loci (Andergassen et al., 2017, 2019; Pandey et al., 2008; Schertzer et al., 2019; Terranova et al., 2008; Vausort et al., 2014).

Notably, half of paternally expressed, ExE-specific imprinted genes appear to depend primarily on G9a-GLP (G9a-GLP $n=12$, Dnmt1 $n=11$, PRC2 $n=1$ ), which include all previously defined H3K27me3-dependent non-canonical imprinted genes 
(Chen et al., 2019; Hanna et al., 2019; Inoue et al., 2017a) (Figure 3A right, Figure 3B bottom, Table S1 sheet J). These results show an interaction between H3K9 and H3K27 methylation and suggest that $\mathrm{H} 3 \mathrm{~K} 9$ me may be the dominant modification for maintaining and possibly establishing non-canonical imprinting. We also see some indication that these non-canonical imprints are distributed in clusters. For example, genes such as Platr4 and 2400006E01Rik are close to the known noncanonical imprinted gene Jade1, suggesting that this pathway may control an entire imprinted cluster on chromosome 3 (Figure 3C top). G9a-GLP dependent cluster regulation was also observed for the non-canonical imprinted genes Slc38a4 and Gm32885 on chromosome 15 (Figure 3C bottom). Finally, G9a-GLP regulation was also recognized for the paternally expressed Sfmbt2 gene, including one of the genome's largest miRNA clusters.

\section{G9a recognizes ERV-driven promoter elements}

A recent study reported that the long terminal repeats (LTR) of ERV elements can act as alternative promoters for non-canonical imprinted genes and found that maternally-inherited $\mathrm{H} 3 \mathrm{~K} 27 \mathrm{me} 3$ transitions to DNA methylation-based silencing in extraembryonic tissues (Hanna et al., 2019). To examine the effects of G9a-GLP on these elements, we examined the DNA methylation dynamics of our G9a-sensitive genes using published whole-genome bisulfite sequencing (WGBS) data in regulator disrupted E6.5 ExE ( $\Delta$ Dnmt1, $\Delta R n f 2, \Delta$ Eed and $\Delta G 9 a$ ) (Grosswendt et al., 2020) as well as newly generated data for our G9a-GLP double KO (Figure 3C). Strikingly, all of our identified non-canonical imprinted genes show local loss of DNA methylation around their transcription start sites (TSSs) in G9a KOs, but not for either PRC mutant (Figure 3C, Figure S3B). Furthermore, within these imprinted loci, every G9a-dependent differentially methylated region (DMR) overlaps an ERV LTR (Figure 3C, Figure S3B). This finding further strengthens our prior RNA-seq based observation that $\mathrm{H} 3 \mathrm{~K} 9 \mathrm{me}$ appears crucial for non-canonical imprinting and suggests that G9a alone is sufficient to recruit DNA methylation in this context. Next, we investigated whether G9a regulation of ERV-driven promoters is a general regulatory mechanism or specific for non-canonical imprinted regions. To address this question, we first defined ExE DMRs using WT and $\triangle$ G9a WGBS data (see Methods) and identified 3392 hypomethylated (91.8\%) and 299 hypermethylated (8.1\%) DMRs (Figure 3D, Table S1 sheet K). The highest enrichment for hypomethylated DMRs over background was observed for promoters $\left(P=4 \times 10^{-17}\right.$, odds-ratio $=1.5,+/-1 \mathrm{~kb}$ TSS) and LTRs $\left(P=7.9 \times 10^{-50}\right.$, odds-ratio=1.6). In summary, we find that G9a is the critical regulator for ERV-driven non-canonical imprinting and propose that this could be a more general gene regulatory mechanism.

\section{Epigenetic regulation of the inactive $X$ chromosome in females}

We next leveraged our data to explore the architecture of $X$ inactivation as it is regulated by distinct epigenetic pathways. Out of 180 maternally expressed $X$-linked ExE genes (informative in all investigated ExE samples), 114 (63.4\%) change their allelic expression in at least one epigenetic regulator mutant. These are almost entirely explained by PRC-based regulation, for which PRC2 is dominant: 74 X-linked 
genes are derepressed exclusively in $\Delta E$ Ed embryos (65\%), while another 35 genes are shared between $\Delta$ Rnf2 and $\Delta$ Eed (30.7\%) (Figure 4A). This synergy between $\mathrm{PRC} 1$ and PRC2, together with previous reports that maternal EED is sufficient to initiate and establish imprinted $\mathrm{XCl}$ (Harris et al., 2019), confirms that Polycombbased repression is critical for propagating chromosome-wide epigenetic suppression. To learn more about the remaining 66 regulator independent genes (36.6\%), we plotted allelic ratios according to their genomic position across the $X$ chromosome. PRC-independent genes exist in defined clusters and appear to be independent of WT expression levels (Figure 4B, Figure S4A). We, therefore, hypothesized that these clusters might represent initial Xist target loci, for which imprinted Xist expression (and localization) is sufficient to maintain paternal silencing. To explore this, we investigated published Xist RNA Antisense Purification (RAP) data, a method that maps IncRNA interactions with chromatin (Engreitz et al., 2013) as well as H3K27me3 and H2AK119ub ChIP-seq specific for the inactive $X$ chromosome (Żylicz et al., 2019). Our analysis shows a strong enrichment for Xist binding, H3K27me3, and H2AK119ub at PRC-independent loci and an inverse correlation between direct Xist binding and PRC dependence (Figure 4C). Taken together, these findings highlight a central role for both PRCs in translating local Xist recruitment to stably silence the surrounding area.

\section{Three distinct mechanisms for encoding parental-specific gene regulation}

Our results allowed us to build a complete inventory of genes that exhibit parentalspecific expression in the early embryo and placenta and evaluate their dependencies on key epigenetic pathways. From our perturbation data, we are able to assign the regulation of every imprinted region in Epiblast $(n=13)$ and ExE $(n=$ 20 ) to one of three distinct epigenetic mechanisms (Figure 5A). First, known canonical ICRs are established and maintained by germline DMRs. Second, paternally unmethylated ICRs enable IncRNA transcription, which then recruits repressive machinery to distal target genes in cis. Third, a G9a dominant mechanism controls ERV-driven non-canonical imprinting in extraembryonic tissues at genes that are often clustered together. Of the thirteen regulator-dependent imprinted regions in the embryonic lineage, all are associated with gDMRs and primarily represent ICRs (Figure 5B): eleven regions are directly linked to gDMRs, while two regions, Zdbf2 and Slc38a4, translate gDMR information in cis via PRC2, with G9a acting as a secondary mechanism (Auclair et al., 2016; Greenberg et al., 2017). Lastly, the Kcnq1 region directs DNMTs via the IncRNA Kcnq1ot1 to silence the distant gene Phlda2, in line with a previous report (Mohammad et al., 2010).

The extraembryonic lineage shares regulation for the majority of these ICRregulated regions $(n=11$ ) but includes additional ICR-controlled IncRNA clusters, as well as seven non-canonical G9a regulated imprinted regions (Figure $5 \mathbf{C}$ ). Two IncRNA controlled clusters are regulated by Kcnq1ot1 and Airn and incorporate known and novel secondary targets, all located in previously defined extraembryonicspecific silencing domains (Andergassen et al., 2017, 2019; Schertzer et al., 2019). For both clusters, we observe strong dependency for PRC and, to a lesser degree, for $\mathrm{G9a}$, in line with previous reports where these regulators establish repressive 
chromatin at target loci (Nagano et al., 2013; Pandey et al., 2008; Terranova et al., 2008; Wagschal et al., 2008). The G9a-dominant imprinted regions include known and novel non-canonical imprinted genes and mostly lie in defined clusters, such as the Smbt2, Jade1, and Slc38a4 imprinted regions and ERV-driven gene loci (Figure 5C). Finally, our data provide an improved understanding of imprinted $\mathrm{XCl}$, including IncRNA Xist-based PRC2 recruitment that is most important for maintaining suppression at regions distal to primary Xist targets (Figure 5D).

\section{DISCUSSION}

In this study, we systematically perturbed multiple repressive pathways in highly polymorphic embryos to investigate the scope of epigenetically maintained parental imprinting. We find that most canonically imprinted genes are shared between the embryonic and extraembryonic lineages. In contrast, non-canonical imprinting is largely restricted to the extraembryonic lineage. For instance, we identify a new H3K9 methylation-based mechanism that suppresses maternal ERV-driven promoters, which could also represent a more general strategy for gene regulation outside of imprinting. Previously, oocyte-specific H3K27me3 was described as a mechanism for non-canonical imprinting within preimplantation embryos (Inoue et al., 2017c). However, our systematic investigation across multiple different regulators finds only a slight effect in zygotic PRC2 mutants on the allelic ratios of these targets, but complete loss of imprinting and associated reactivation in G9a mutants, suggesting $\mathrm{H} 3 \mathrm{~K} 9 \mathrm{me}$ is critical for establishing non-canonical imprinting.

We also provided mechanistic insides of long-range silencing by imprinted IncRNAs in extraembryonic lineages. Previous reports observed that Kcnq1ot1 and Airn are expressed from the zygote stage and deployed to local targets to recruit PRCs (Andergassen et al., 2017, 2019; Nagano et al., 2008; Schertzer et al., 2019; Terranova et al., 2008). Notably, we find that Dnmt1 KO embryos activate the silent Kcnq1ot1 allele but do not affect imprinted expression of its PRC2-dependent targets. To capture both forms of deregulation independently, a transient window in preimplantation may allow IncRNAs to relay germline ICR information to their genetic neighborhood before DNA methylation is lost. As such, both DNA methylationspecific ICRs and their secondary, PRC2-dependent targets must be established very soon after fertilization. This model would also explain why Dnmt1-dependent imprints are shared between embryonic and extraembryonic lineages. Finally, Kcnq1ot1 targets in PRC mutants show biallelic expression, hinting that oocytespecific PRC is sufficient to initiate imprinted silencing, but zygotic PRC is required to maintain it. A similar mechanism may apply for the novel maternally expressed imprinted genes Pnldc1 and $T$ that lie within the IncRNA Airn silencing domain, although Airn itself is only lowly expressed during this developmental period (Andergassen et al., 2017, 2019; Schertzer et al., 2019).

Furthermore, our approach allowed us to shed light on the $\mathrm{XCI}$ maintenance in vivo. We elucidated both PRCs as critical factors that maintain portions of the inactive $X$ chromosome in a repressed state, while perturbing of DNA methylation or H3K9me pathways had no impact. Finally, we identified PRC-independent gene clusters on the $X$ chromosome, which resembles the early binding sites of Xist- 
mediated suppression. This would suggest that different territories on the $X$ are uniquely dependent on direct binding via Xist or translate this local cue to distal areas by Polycomb. Together, our data provide a comprehensive inventory of the epigenetic mechanisms of parental-specific imprinting, which is also fundamental for many X-linked diseases as well as imprinting disorders where unlocking the silent healthy allele is an attractive therapeutic strategy.

Notably, our study provides a platform for future investigation into the molecular genetics of parental imprinting and $X$ inactivation by combining genetic perturbation with fertilization using highly polymorphic strains. For example, we establish that non-canonical imprinting requires G9a to maintain maternal silencing of ERV promoter-containing genes but do not yet understand the nature of imprint establishment in the maternal germline. Conditional knockouts in the female germline will further expand our understanding of the relationship between the epigenetic machinery that encodes these imprints and those that interpret them to facilitate maintenance. Similarly, our PRC mutants exhibit limited reactivation of autosomal imprints or of paternal $X$ reactivation, with only some leaky expression, whereas Dnmt1 and G9a exhibited little or no effect. It is possible that these regulators provide additional levels of repression that can only be observed when PRCs are absent. Double or triple regulator mutant strategies would permit an exact, quantitative investigation for each independent regulator's contribution to chromosome-level silencing.

Upon completion of this work, it appears that very few, if any, parent-specific allelic expression cannot be explained with one of the three reported mechanisms described above. However, little is known about how these non-canonical imprints are innovated over evolutionary time or the degree to which they are conserved across eutherian mammals in comparison to classic ICRs. Moreover, their striking enrichment in the placental lineage further highlights this tissue as a domain for expanded epigenetic innovation in mammals. Future studies in these areas will provide greater clarity for the roles these additional imprinting mechanisms play in supporting fetal development. 


\section{FIGURES}



Figure 1 An inventory of parental-specific expression for male and female embryos in early embryonic and extraembryonic lineages

(A) Simplified schematic of the experimental system used to obtain a parent-specific expression landscape (imprinting and X-inactivation). E6.5 Epiblast (light green) and extraembryonic ectoderm (ExE, dark green) are isolated from F1 reciprocal crosses ( $n=11$, BDF1xCAST $4 \mathrm{~m} / 3 f$ and CASTxBDF1 $1 \mathrm{~m} / 3 \mathrm{f}$ ) and subjected to RNA-seq. Colors are used in figures throughout the manuscript to highlight the tissue of origin.

(B-C) Imprinted genes identified in the E6.5 Epiblast and ExE (red: maternally expressed, blue: paternal expressed) using a median imprinted score cutoff and allelic ratios of 1 and 0.25 , respectively (dashed line). Corresponding heatmaps show the imprinted ratios in the forward (BDF1xCAST) and reverse (CASTxBDF1) cross. Novel identified imprinted genes are indicated in white. This also includes the observation of maternalspecific expression of the major regulator Brachyury $(T)$ within the ExE.

(D) Overlap of imprinted genes between E6.5 Epiblast and ExE.

(E) Scatter plot showing the imprinted ratios for X-linked genes in ExE between forward (BDF1xCAST) and reverse (CASTxBDF1) crosses. Maternally expressed genes (red), $\mathrm{XCl}$ escaper genes (green), and strainspecific escape from the CAST (brown) and BDF1 (grey) inactive $X$ chromosome are indicated. The dashed line indicates the escaper cutoff of 0.25 .

(F) Chromosomal overview of genes that maintain biallelic expression on the Xi ("escapers") with shared above and strain-specific below. Asterisk indicates escaper genes that function as chromatin modifiers.



Figure S1

(A) Unsupervised clustering of pairwise Pearson correlation from log-transformed expression levels (TPM) of the forward (BDF1xCAST) and reverse (CASTxBDF1) cross confirms the lineage identity.

(B) Violin plots displaying the allelic ratio of autosomal and X-linked genes from forward and reverse crosses for both genders in the Epiblast and ExE. The black dot highlights the IncRNA Xist. 


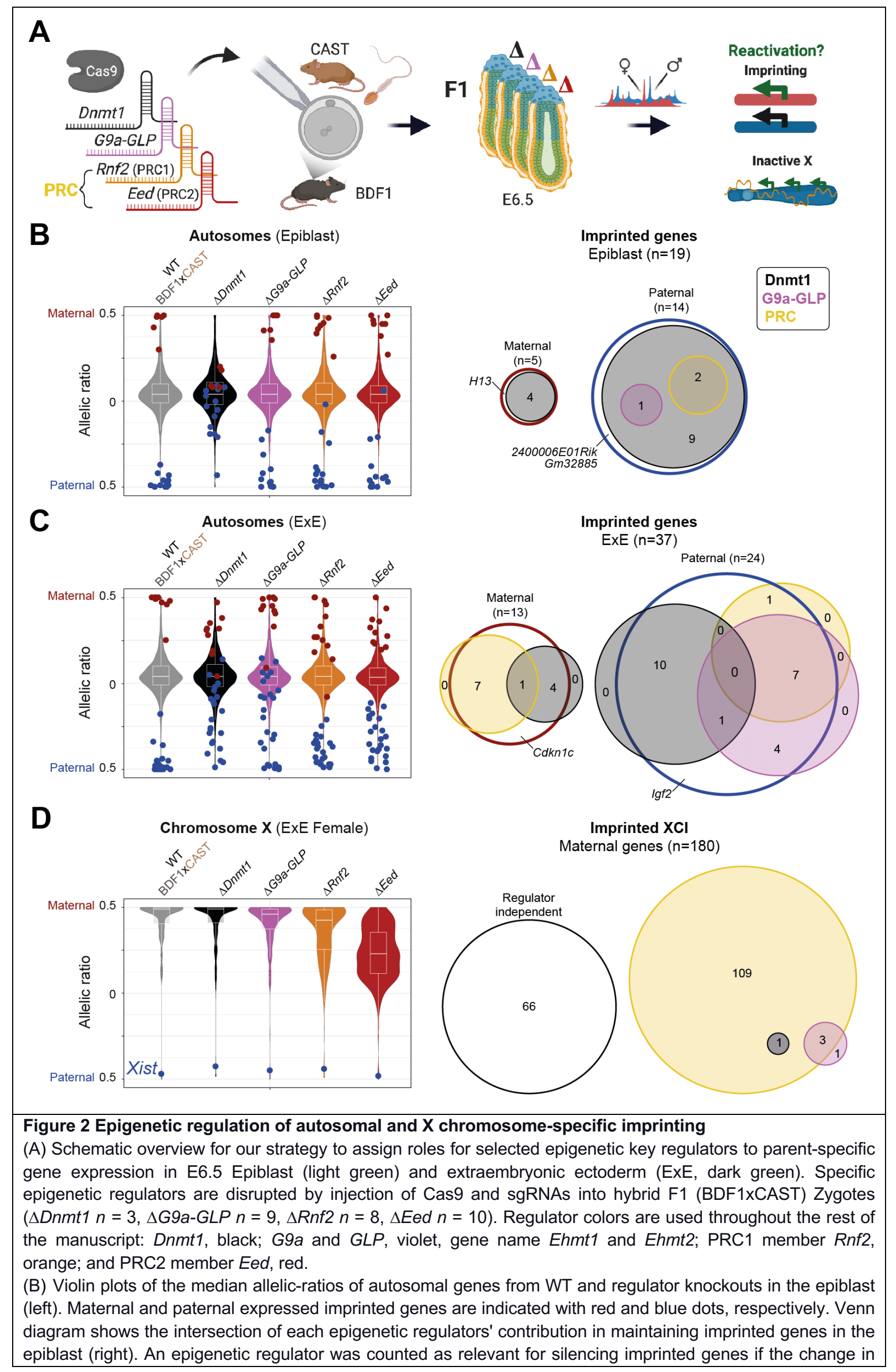


bioRxiv preprint doi: https://doi.org/10.1101/2021.04.30.442087; this version posted April 30, 2021. The copyright holder for this preprint (which was not certified by peer review) is the author/funder, who has granted bioRxiv a license to display the preprint in perpetuity. It is made available under aCC-BY-NC-ND 4.0 International license.

allelic ratio between WT and KO was $\geq 20 \%$. PRC1 and PRC2 were summarized as PRC by using the higher delta. DNA methylation-dependent imprinting is most frequent in the epiblast. Regulator independent imprinted genes have a delta allelic ratio $<20 \%$ in all disrupted regulators. Imprinted genes with less than two informative allelic ratio values in any regulator disruption dataset are not shown.

(C) As in (B) for the ExE lineage. Extraembryonic imprints appear to depend on a more diverse set of regulators.

(D) Violin plots displaying the median allelic-ratio of X-linked genes from WT and regulator knockouts in female ExE (left). The blue dot highlights the allelic ratio of the IncRNA Xist. Successful extraembryonic XCI depends on PRC2 and, to a lesser degree, PRC1 downstream of paternal Xist expression, which is stable in all regulator mutants examined (Figure S2D). Venn diagram for imprinted $\mathrm{XCl}$ (right) as shown for autosomal imprinting in (B-C). An epigenetic regulator was counted as relevant for silencing $X$-linked genes if the delta allelic ratio change between WT and KO for any regulator was $\geq 20 \%$. Regulator independent $X$-linked genes have a delta allelic ratio $<20 \%$ in all disrupted regulators.

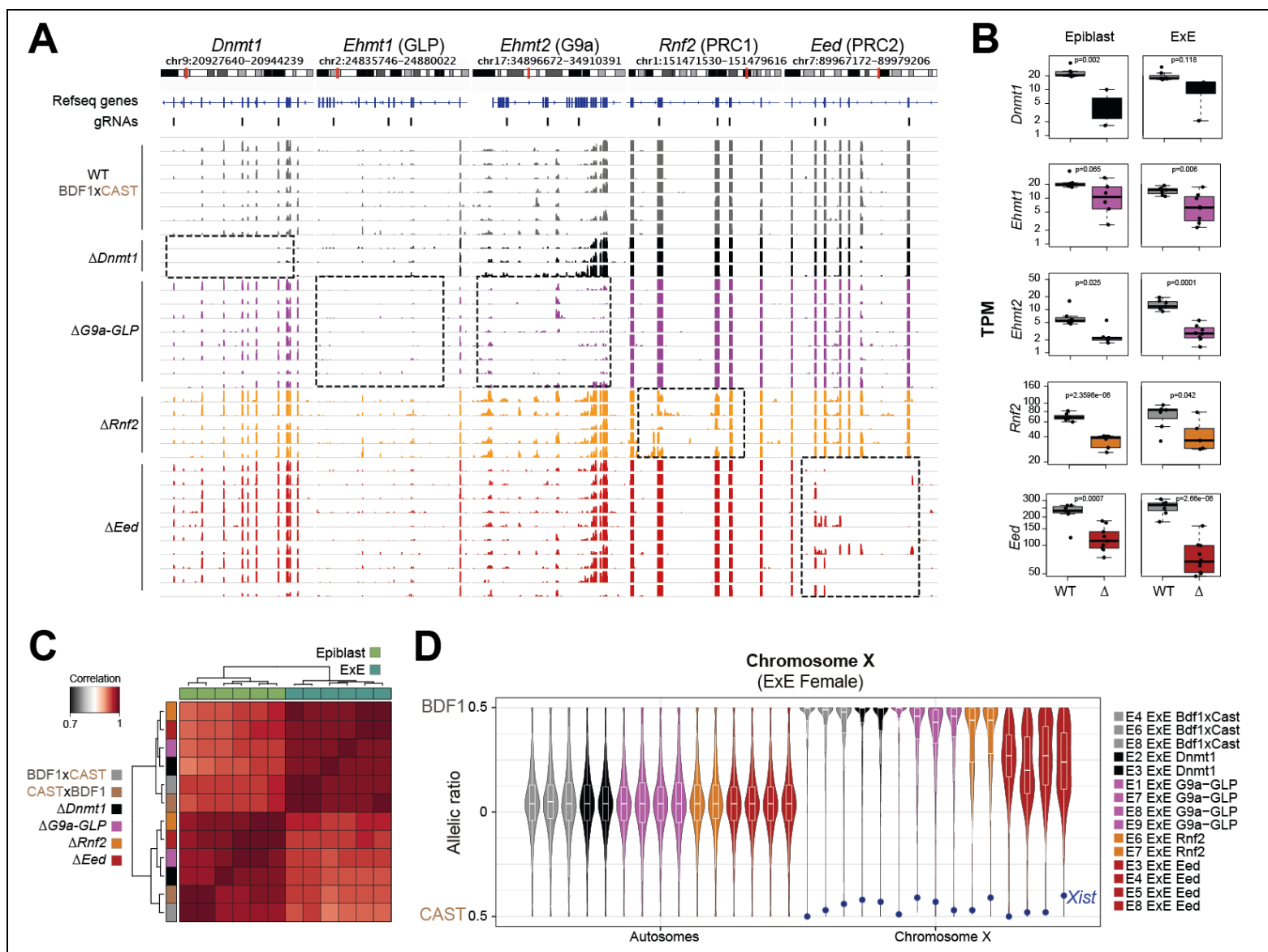

\section{Figure S2}

(A) Genome browser tracks showing the ExE RNA-seq results and gRNA positions of the targeted loci Dnmt1, Ehmt1, Ehmt2, Rnf2, and Eed for wildtype and regulator disrupted embryos.

(B) Boxplot showing the expression levels (TPM) of the targeted loci between wildtype and KO embryos for the Epiblast and ExE lineage.

(C) Unsupervised clustering of pairwise Pearson correlation from wildtype and regulator disrupted samples show broad retention of epiblast and ExE transcriptional signatures (TPM, replicates are combined using the average).

(D) Violin plots displaying the allelic-ratio of X-linked genes from WT and regulator knockouts for every female ExE replicate (left). The blue dot highlights the allelic ratio of the IncRNA Xist. 


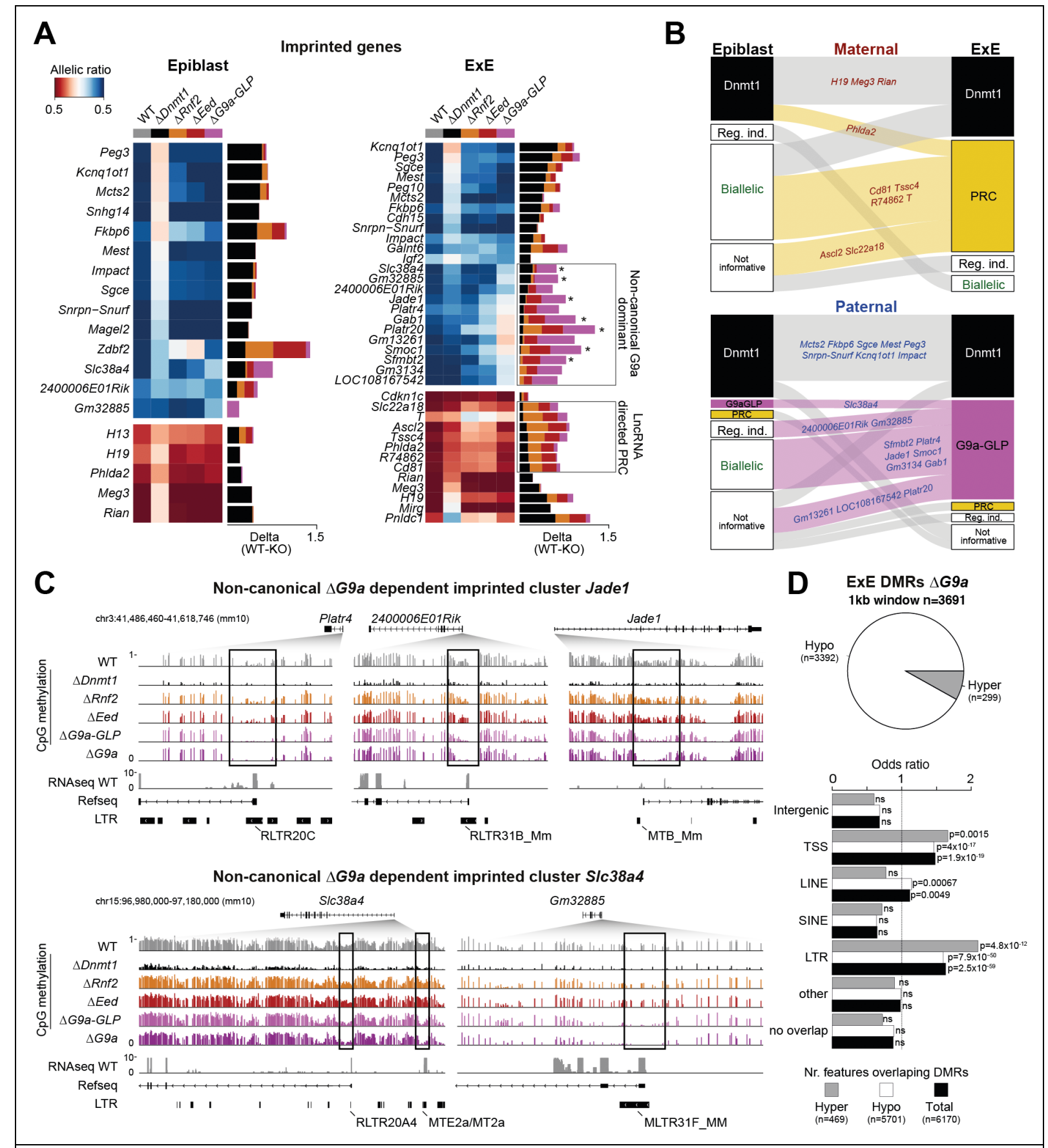

Figure 3 G9a controls endogenous retrovirus promoters of non-canonical imprinted genes

(A) Allelic ratio of imprinted genes and the corresponding delta changes between wildtype (BDF1xCAST) and regulator disruption identified in the Epiblast (left) and ExE (right) lineage. Heatmap ranked by delta allelic ratio change between WT and Dnmt1 deletion. For ExE, boxes highlight DNA methylation independent non-canonical expressed genes (known* and novel) and IncRNA directed PRC targets. Notably, ExE-specific imprinting is most apparent for a set of paternally-expressed, G9a-GLP controlled loci that are only weakly dependent on PRCs. Imprinted genes with less than two informative allelic ratio values in any regulator disruption dataset are not shown.

(B) Flow diagram outlining changes in the imprinted landscape between Epiblast and ExE for maternally (top) and paternally (bottom) expressed genes.

(C) Genome browser tracks for WGBS and RNA-seq data from WT and regulator disrupted E6.5 ExE lineages capturing the two non-canonical G9a dependent imprinted clusters Jade1 (top) and Slc38a4 (bottom). Boxes highlight G9a dependent hypomethylated DMRs (Overlapping ERV LTRs are indicated).

(D) Identified ExE DMRs using WT and $\triangle$ G9a WGBS data (1kb window, $n=3691$, |delta cutoff $\geq 20 \%$ ). Pie chart showing the proportion of hypo- and hypermethylated DMRs (top). Feature enrichment of the identified DMRs over background was calculated for intergenic, genic ( $\pm 1 \mathrm{~kb}$ of TSS), and different repeat classes using the Fisher's exact test (bottom). 
bioRxiv preprint doi: https://doi.org/10.1101/2021.04.30.442087; this version posted April 30, 2021. The copyright holder for this preprint (which was not certified by peer review) is the author/funder, who has granted bioRxiv a license to display the preprint in perpetuity. It is made available under aCC-BY-NC-ND 4.0 International license.

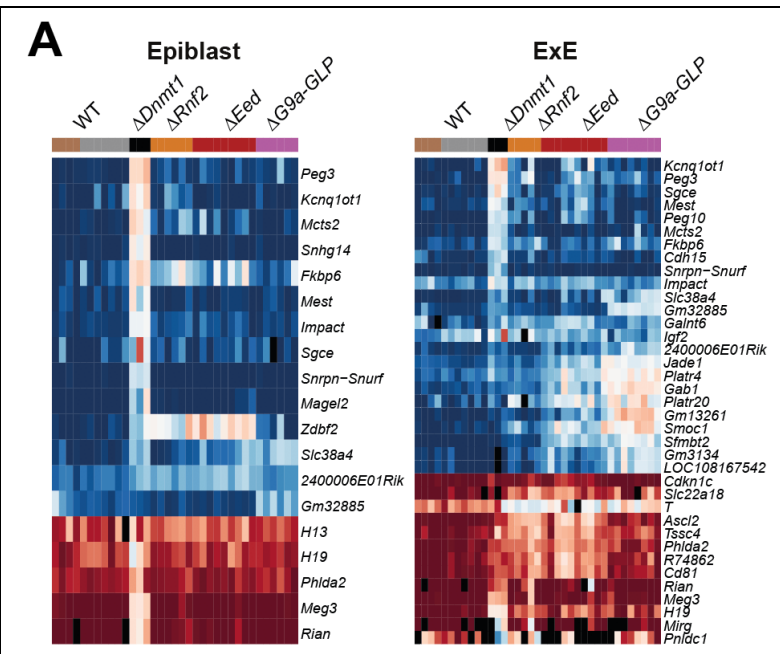

B

Non-canonical $\Delta G 9 a$ dependent regions (ExE)

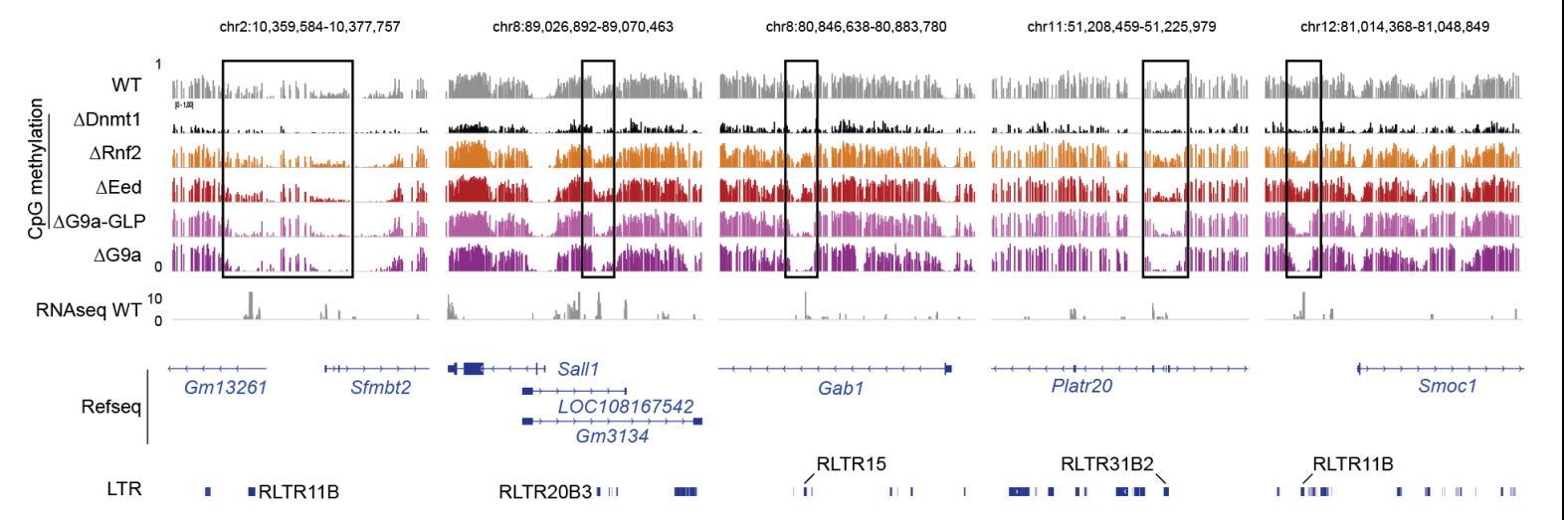

Figure S3

(A) Allelic ratio of imprinted genes between wildtype (BDF1xCAST) and regulator disruption identified in the epiblast (left) and ExE (right) lineage for each embryo. Heatmap ranked by delta allelic ratio change between WT and Dnmt1 deletion. The black color indicates non-informative allelic ratios for a given gene and replicate.

(B) Genome browser tracks for WGBS and RNA-seq data from WT and regulator disrupted E6.5 ExE lineages capturing the non-canonical G9a dependent imprinted loci Gm13261, Sfmbt2, LOC108167542, Gm3134, Gab1, Platr20, and Smoc1. Boxes highlight G9a dependent hypomethylated DMRs (Overlapping ERV LTRs are indicated). 

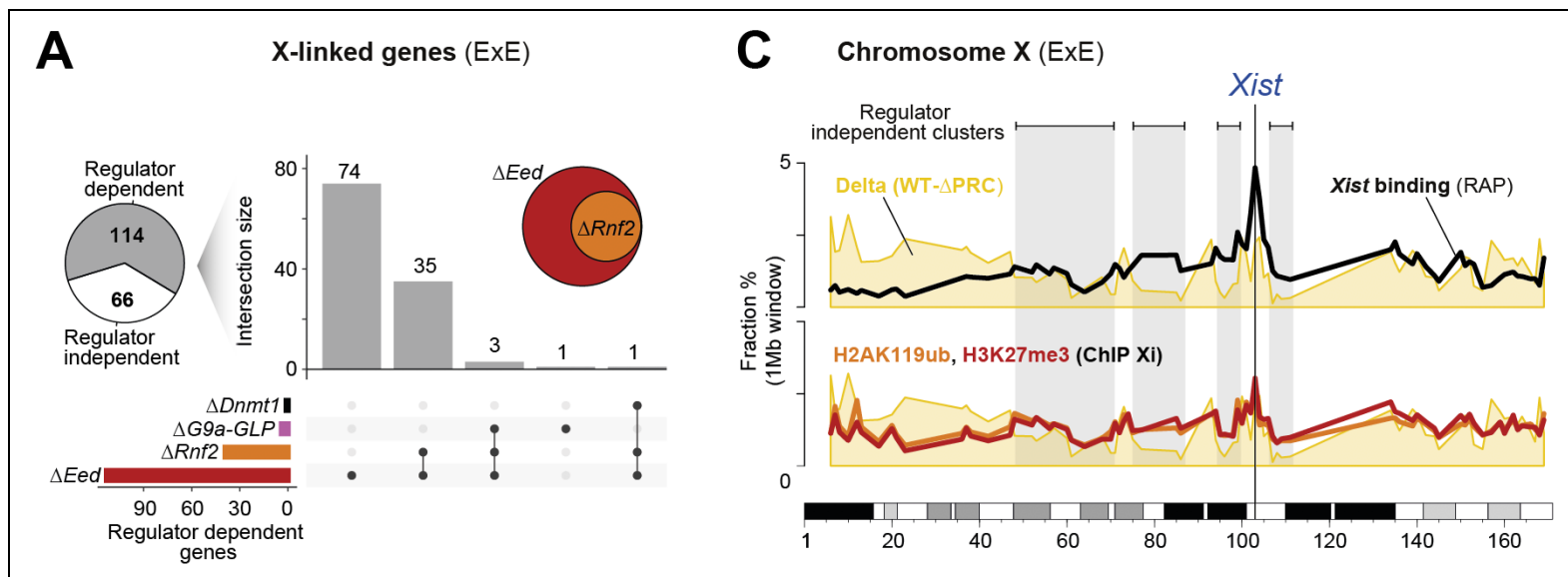

B

Chromosome X (ExE) Xist

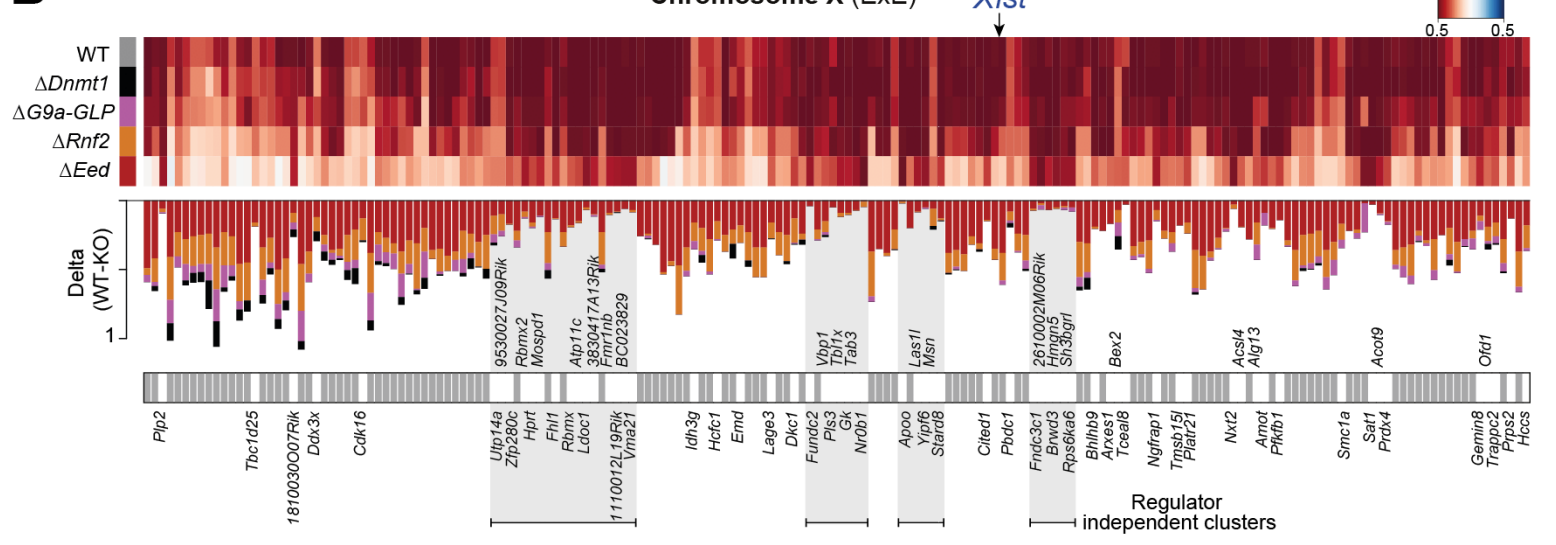

Figure 4 Polycomb-based repression is critical for the maintenance of the imprinted $\mathrm{X}$ chromosome

(A) Pie chart showing the proportion of regulator dependent or independent genes for imprinted XCl. A gene was called regulator dependent if the delta allelic ratio changes between WT and $K O \geq 20 \%$ for any regulator. UpSet plot shows the overlap across the four disrupted regulator members: Dnmt1, G9a-GLP, Rnf2, and Eed. The inset Venn diagram highlights the high overlap between Eed and Rnf2 dependent X-linked genes. Genes that escape imprinted $\mathrm{XCl}$ were excluded from this analysis

(B) Regulator-independent X-linked genes are organized into distinct spatial clusters. Allelic ratios of maternally expressed X-linked genes and the corresponding delta changes between wildtype (BDF1xCAST) and regulator disrupted E6.5 ExE lineages are shown (X-linked gene Matrix ranked by the genomic position). Regulatorindependent $X$-linked genes are indicated. A gene was called regulator independent if the delta allelic ratio changes between WT and $\mathrm{KO}<20 \%$ for every regulator.

(C) Regulator independent regions occur in domains with high Xist enrichment and repressive chromatin. $1 \mathrm{Mb}$ windows summarizing (average) the X-linked gene delta changes between WT and PRC, for Xist enrichment over input (RAP) (Engreitz et al., 2013) and for H3K27me3 and H2AK119ub enrichment on the inactive X (Żylicz et al., 2019) across the entire X-chromosome.

A X-linked genes (ExE)

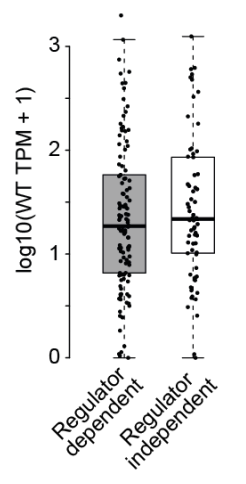

Figure S4

(A) Wildtype expression level (TPM) of regulator dependent and independent X-linked genes in ExE. 


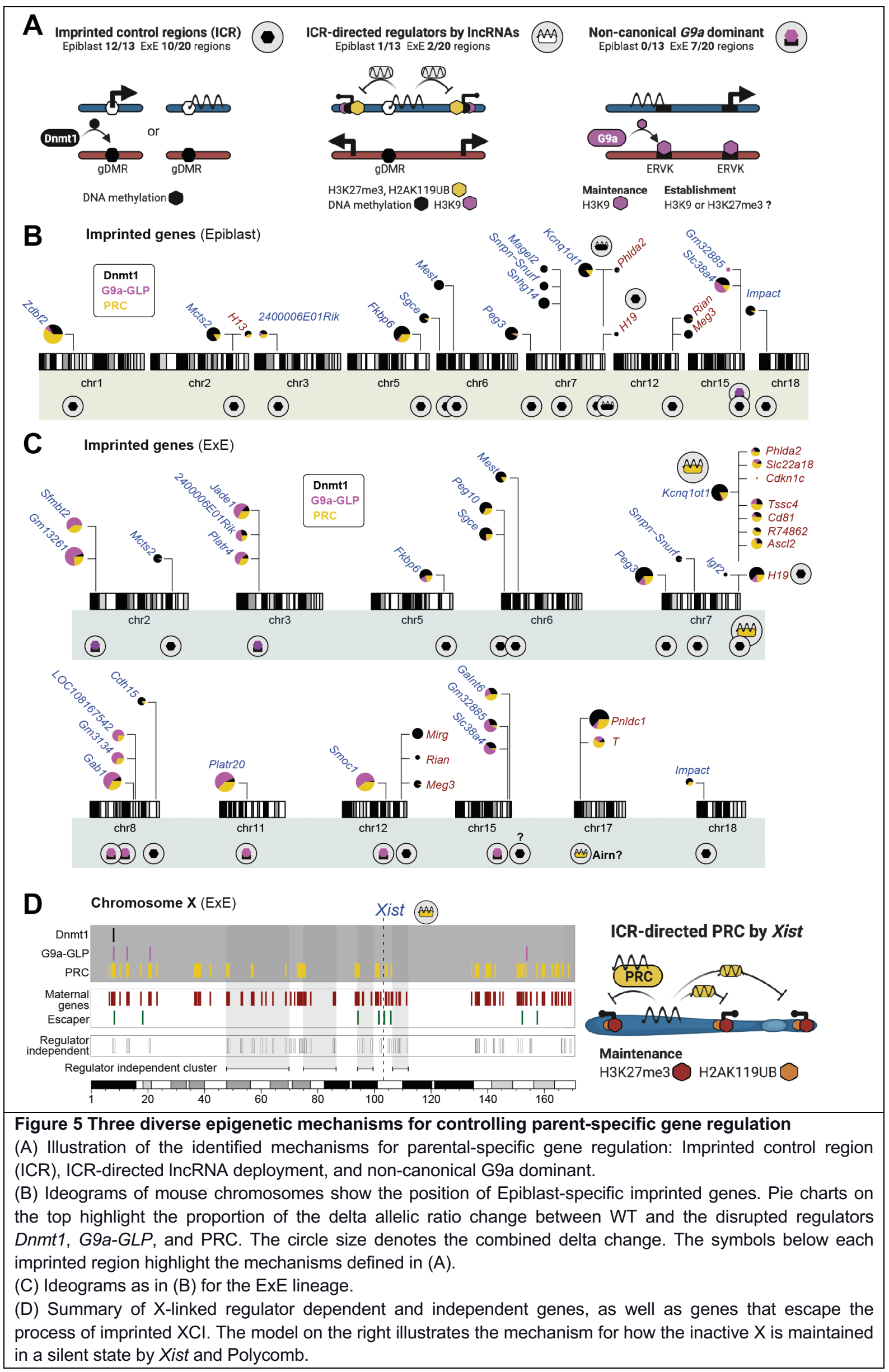


bioRxiv preprint doi: https://doi.org/10.1101/2021.04.30.442087; this version posted April 30, 2021. The copyright holder for this preprint (which was not certified by peer review) is the author/funder, who has granted bioRxiv a license to display the preprint in perpetuity. It is made available under aCC-BY-NC-ND 4.0 International license.

\section{REFERENCES}

1. Andergassen, D., Dotter, C.P., Kulinski, T.M., Guenzl, P.M., Bammer, P.C., Barlow, D.P., Pauler, F.M., and Hudson, Q.J. (2015). Allelome.PRO, a pipeline to define allele-specific genomic features from high-throughput sequencing data. Nucleic Acids Res. 43.

2. Andergassen, D., Dotter, C.P., Wenzel, D., Sigl, V., Bammer, P.C., Muckenhuber, M., Mayer, D., Kulinski, T.M., Theussl, H.-C., Penninger, J.M., et al. (2017). Mapping the mouse Allelome reveals tissue-specific regulation of allelic expression. Elife 6, 1-29.

3. Andergassen, D., Muckenhuber, M., Bammer, P.C., Kulinski, T.M., Theussl, H.-C., Shimizu, T., Penninger, J.M., Pauler, F.M., and Hudson, Q.J. (2019). The Airn IncRNA does not require any DNA elements within its locus to silence distant imprinted genes. PLOS Genet. 15, e1008268.

4. Anders, S., Pyl, P.T., and Huber, W. (2015). HTSeq--a Python framework to work with high-throughput sequencing data. Bioinformatics 31, 166-169.

5. Auclair, G., Borgel, J., Sanz, L.A., Vallet, J., Guibert, S., Dumas, M., Cavelier, P., Girardot, M., Forné, T., Feil, R., et al. (2016). EHMT2 directs DNA methylation for efficient gene silencing in mouse embryos. Genome Res. 26, $192-202$.

6. Barlow, D.P., and Bartolomei, M.S. Genomic Imprinting in Mammals.

7. Calaway, J.D., Lenarcic, A.B., Didion, J.P., Wang, J.R., Searle, J.B., McMillan, L., Valdar, W., and Pardo-Manuel de Villena, F. (2013). Genetic Architecture of Skewed X Inactivation in the Laboratory Mouse. PLoS Genet.

8. Chadwick, L.H., Pertz, L.M., Broman, K.W., Bartolomei, M.S., and Willard, H.F. (2006). Genetic control of X chromosome inactivation in mice: Definition of the Xce candidate interval. Genetics 173, 2103-2110.

9. Chen, Z., Yin, Q., Inoue, A., Zhang, C., and Zhang, Y. (2019). Allelic H3K27me3 to allelic DNA methylation switch maintains noncanonical imprinting in extraembryonic cells. Sci. Adv. 5, eaay7246.

10. Chenoweth, J.G., and Tesar, P.J. (2010). Isolation and maintenance of mouse epiblast stem cells. Methods Mol. Biol. 636, $25-44$.

11. Dobin, A., Davis, C.A., Schlesinger, F., Drenkow, J., Zaleski, C., Jha, S., Batut, P., Chaisson, M., and Gingeras, T.R. (2013). STAR: ultrafast universal RNA-seq aligner. Bioinformatics 29, 15-21.

12. Engreitz, J.M., Pandya-Jones, A., McDonel, P., Shishkin, A., Sirokman, K., Surka, C., Kadri, S., Xing, J., Goren, A., Lander, E.S., et al. (2013). The Xist IncRNA exploits three-dimensional genome architecture to spread across the X chromosome. Science 341 , 1237973.

13. Greenberg, M.V.C., Glaser, J., Borsos, M., Marjou, F. El, Walter, M., Teissandier, A., and Bourc'his, D. (2017). Transient transcription in the early embryo sets an epigenetic state that programs postnatal growth. Nat. Genet. 49, 110-118.

14. Grosswendt, S., Kretzmer, H., Smith, Z.D., Kumar, A.S., Hetzel, S., Wittler, L., Klages, S., Timmermann, B., Mukherji, S., and Meissner, A. (2020). Epigenetic regulator function through mouse gastrulation. Nature 584, 102-108.

15. Hanna, C.W., Pérez-Palacios, R., Gahurova, L., Schubert, M., Krueger, F., Biggins, L., Andrews, S., Colomé-Tatché, M., Bourc'His, D., Dean, W., et al. (2019). Endogenous retroviral insertions drive non-canonical imprinting in extra-embryonic tissues. Genome Biol. 20, 225.

16. Harris, C., Cloutier, M., Trotter, M., Hinten, M., Gayen, S., Du, Z., Xie, W., and Kalantry, S. (2019). Conversion of random Xinactivation to imprinted $\mathrm{x}$-inactivation by maternal prc2. Elife 8.

17. Inoue, A., Jiang, L., Lu, F., Suzuki, T., and Zhang, Y. (2017a). Maternal H3K27me3 controls DNA methylation-independent imprinting. Nature.

18. Inoue, A., Jiang, L., Lu, F., and Zhang, Y. (2017b). Genomic imprinting of Xist by maternal H3K27me3. Genes Dev. 31, $1927-1932$.

19. Inoue, A., Jiang, L., Lu, F., Suzuki, T., and Zhang, Y. (2017c). Maternal H3K27me3 controls DNA methylation-independent imprinting. Nature 547, 419-424.

20. Jiang, Q., Ang, J.Y.J., Lee, A.Y., Cao, Q., Li, K.Y., Yip, K.Y., and Leung, D.C.Y. (2020). G9a Plays Distinct Roles in Maintaining DNA Methylation, Retrotransposon Silencing, and Chromatin Looping. Cell Rep. 33, 108315.

21. Keane, T.M., Goodstadt, L., Danecek, P., White, M.A., Wong, K., Yalcin, B., Heger, A., Agam, A., Slater, G., Goodson, M., et al. (2011). Mouse genomic variation and its effect on phenotypes and gene regulation. Nature 477, $289-294$.

22. Labun, K., Montague, T.G., Gagnon, J.A., Thyme, S.B., and Valen, E. (2016). CHOPCHOP v2: a web tool for the next generation of CRISPR genome engineering. Nucleic Acids Res. 44, W272-W276.

23. Mohammad, F., Mondal, T., Guseva, N., Pandey, G.K., and Kanduri, C. (2010). Kcnq1ot1 noncoding RNA mediates transcriptional gene silencing by interacting with Dnmt1. Development 137, 2493-2499.

24. Monk, D., Mackay, D.J.G., Eggermann, T., Maher, E.R., and Riccio, A. (2019). Genomic imprinting disorders: lessons on how genome, epigenome and environment interact. Nat. Rev. Genet. 20, 235-248.

25. Nagano, T., Mitchell, J.A., Sanz, L.A., Pauler, F.M., Ferguson-Smith, A.C., Feil, R., and Fraser, P. (2008). The Air noncoding RNA epigenetically silences transcription by targeting G9a to chromatin. Science (80-. ). 322, 1717-1720.

26. Nagano, T., Lubling, Y., Stevens, T.J., Schoenfelder, S., Yaffe, E., Dean, W., Laue, E.D., Tanay, A., and Fraser, P. (2013). Singlecell Hi-C reveals cell-to-cell variability in chromosome structure. Nature 502, 59-64.

27. Pandey, R.R., Mondal, T., Mohammad, F., Enroth, S., Redrup, L., Komorowski, J., Nagano, T., Mancini-DiNardo, D., and Kanduri, C. (2008). Kcnq1ot1 Antisense Noncoding RNA Mediates Lineage-Specific Transcriptional Silencing through Chromatin-Level Regulation. Mol. Cell 32, 232-246.

28. Schertzer, M.D., Braceros, K.C.A., Starmer, J., Cherney, R.E., Lee, D.M., Salazar, G., Justice, M., Bischoff, S.R., Cowley, D.O., Ariel, P., et al. (2019). IncRNA-Induced Spread of Polycomb Controlled by Genome Architecture, RNA Abundance, and CpG Island DNA. Mol. Cell 75, 523-537.e10.

29. Smith, Z.D., Shi, J., Gu, H., Donaghey, J., Clement, K., Cacchiarelli, D., Gnirke, A., Michor, F., and Meissner, A. (2017). Epigenetic restriction of extraembryonic lineages mirrors the somatic transition to cancer. Nature 549, 543-547.

30. Terranova, R., Yokobayashi, S., Stadler, M.B., Otte, A.P., van Lohuizen, M., Orkin, S.H., and Peters, A.H.F.M. (2008). Polycomb Group Proteins Ezh2 and Rnf2 Direct Genomic Contraction and Imprinted Repression in Early Mouse Embryos. Dev. Cell 15, 668679.

31. Tucci, V., Isles, A.R., Kelsey, G., Ferguson-Smith, A.C., Bartolomei, M.S., Benvenisty, N., Bourc'his, D., Charalambous, M., Dulac, C., Feil, R., et al. (2019). Genomic Imprinting and Physiological Processes in Mammals. Cell 176, 952-965.

32. Vausort, M., Wagner, D.R., and Devaux, Y. (2014). Long noncoding RNAs in patients with acute myocardial infarction. Circ. Res. $115,668-677$.

33. Wagschal, A., Sutherland, H.G., Woodfine, K., Henckel, A., Chebli, K., Schulz, R., Oakey, R.J., Bickmore, W.A., and Feil, R. (2008). G9a Histone Methyltransferase Contributes to Imprinting in the Mouse Placenta. Mol. Cell. Biol. 28, 1104-1113.

34. Wang, H., Yang, H., Shivalila, C.S., Dawlaty, M.M., Cheng, A.W., Zhang, F., and Jaenisch, R. (2013). One-Step Generation of Mice Carrying Mutations in Multiple Genes by CRISPR/Cas-Mediated Genome Engineering. Cell 153, 910-918.

35. Żylicz, J.J., and Heard, E. (2020). Molecular Mechanisms of Facultative Heterochromatin Formation: An X-Chromosome Perspective. Annu. Rev. Biochem. 89, 255-282.

36. Żylicz, J.J., Bousard, A., Žumer, K., Dossin, F., Mohammad, E., da Rocha, S.T., Schwalb, B., Syx, L., Dingli, F., Loew, D., et al. (2019). The Implication of Early Chromatin Changes in X Chromosome Inactivation. Cell 176, 182-197.e23. 


\section{METHOD DETAILS}

\section{Mouse strains}

All procedures have been performed in our specialized facility, followed all relevant animal welfare guidelines and regulations, and were approved by Harvard University IACUC protocol (28-21). CAST/EiJ (CAST) and B6D2F1/J (BDF1) mice were purchased from the Jackson Laboratory.

\section{Disrupting epigenetic regulators in F1 background using zygotic CRISPR-Cas9 injection}

Embryos were generated as previously described (Wang et al., 2013). Briefly, BDF1 strain female mice (age 6-8 weeks, Jackson Labs) were superovulated by serial Pregnant Mare Serum Gonadotropin (5 IU per mouse, Prospec Protein Specialists) and human chorionic gonadotropin (5 IU, Millipore) injections $46 \mathrm{~h}$ apart. The following day, MII stage oocytes were isolated in M2 media supplemented with hyaluronidase (Millipore) and stored in $25 \mu \mathrm{l}$ drops of pre-gassed KSOM with halfstrength concentration of amino acids (Millipore) under mineral oil (Irvine Scientific). Zygotes were generated by piezo-actuated intracytoplasmic sperm injection (ICSI. See Ref. (Grosswendt et al., 2020)) using thawed CAST strain sperm in batches of 30-50 oocytes and standard micromanipulation equipment, including a Hamilton Thorne XY Infrared laser, Eppendorf Transferman NK2 and Patchman NP2 micromanipulators, and a Nikon Ti-U inverted microscope. Alternatively, for the reciprocal cross, BDF1 males were naturally mated to CAST females and screened for copulation plugs, after which E6.5 stage embryos were isolated accordingly, with the date of the copulation plug score day E0.5.

For zygotic disruption, pronuclear stage 3 (PN3) zygotes were injected with $200 \mathrm{ng} \mathrm{ul}^{-1}$ Cas $9 \mathrm{mRNA}$ and a $100 \mathrm{ng} \mathrm{ul}^{-1}$ equimolar ratio of 3-4 sgRNAs targeting different exons of an epigenetic regulator gene locus (designed using ChopChop (Labun et al., 2016) and the IDT CRISPR-Cas9 guide RNA checker, as previously described in Ref. (Smith et al., 2017)). Injections utilized the same microinjection setup and Piezo-actuated injection of front-filled 6-7 $\mu \mathrm{m}$ injection needles. At around $84 \mathrm{~h}$ after fertilization, cavitated blastocysts were transferred into the uterine horns of pseudopregnant CD-1 strain females (25-35g, Charles River) generated by mating with vasectomized SW strain males (Taconic), which results in a $24 \mathrm{~h}$ offset in gestational time to accommodate implantation, after which animals were monitored for 5 days for embryo isolation at E6.5.

\section{Embryo isolation and Library preparation}

At E6.5, animals were euthanized and the uterine horn removed. Purified epiblast and ExE were isolated according to Ref. (Chenoweth and Tesar, 2010) with a few modifications. Briefly, E6.5 embryos were removed from deciduae and transferred to independent $25 \mu \mathrm{L}$ drops of M2 media. Using a glass flame-pulled capillary, Reichart's membrane was reflected, and the embryo carefully bisected along the epiblast, ExE boundary. Then, each Epiblast/ExE pair was transferred into an individual drop of dissociation medium containing $0.5 \%$ trypsin and $2.5 \%$ pancreatin 
in PBS (w/v, Sigma). Embryos were cultured with slow orbital rotation at $4^{\circ} \mathrm{C}$ for 15 minutes, after which they were transferred into new M2 drops. After $\sim 5$ minutes of resting, Epiblast and ExE were passed through a slightly narrower flame-pulled glass capillary to remove the visceral endoderm without disrupting the target tissue. Finally, each tissue was serially washed in $0.1 \%$ BSA in PBS prior to snap freezing in lysis buffer.

Approximately 300-600 cells were collected from E6.5 embryos and directly transferred to $2.6 \mu$ l of Lysis Buffer (Takara Bio USA, Inc.) followed by snap-freezing at $-80^{\circ} \mathrm{C}$ in preparation for CDNA synthesis using the SMART-Seq v4 assay. Fulllength cDNA was prepared using the SMART-Seq v4 Ultra Low Input RNA Kit for Sequencing and sequencing libraries prepared using the Nextera XT DNA library preparation kit (Illumina). The resulting libraries were evaluated using a 4200 TapeStation (Agilent Technologies) and quantified by qPCR. Libraries were pooled and sequenced on an Illumina NovaSeq SP or S1 flow cell using paired-end, 50 bp reads.

\section{RNA-seq and allele-specific analysis}

The RNA-seq data were aligned to the mm10 reference genome using the STAR aligner (Dobin et al., 2013) (STAR version 2.5.0 c: -outFilterMultimapNmax 1). The read counts for every RefSeq isoform (RefSeq gene annotation (downloaded February 2018) were determined using the htseq-count phyton script (Anders et al., 2015) (version 0.6.1) and further normalized in $R$.

The Allelome.PRO approach was used to calculate allele-specific expression from the RNA-seq data as outlined in (Andergassen et al., 2015). Briefly, the Allelome.PRO pipeline uses strain-specific single-nucleotide polymorphisms (SNPs) to assign RNA-seq reads to the corresponding allele in F1 crosses. To obtain allelespecific ratios and scores from a single replicate, we slightly modified the pipeline (We will upload the modified Allelome.PRO version on SourceForge upon paper acceptance: https://sourceforge.net/projects/allelomepro/). The Allelome.PRO approach requires a gene and SNP annotation:

For the gene annotation, we used RefSeq, including 35856 genes. To avoid unreliable allele-specific calls, we modified the annotation for known overlapping imprinted genes: First, we removed all the isoforms of H13, Kcnq1, and Copg2 overlapping the genes Mcts2, Kcnq1ot1, and Mest2, respectively. Second, we removed the two genes Peg3os and Gm33149, which overlap the known imprinted genes Peg3 and Gm32885, respectively. Third, we truncated the gene Tsix to avoid overlap with Xist and Gm32061 to prevent overlap with the two imprinted genes Ndn and Magel2. Finally, we assigned the gene name Snrpn-Snurf to the Snrpn and Snurf isoforms since both belong to the same gene.

To generate the SNP annotation for our F1 crosses between CAST and BDF1 (BDF1: F1 cross between C57BL/6J female x DBA/2J), we first derived 20,606,390 high confidence SNPs between CAST and C57BL/6J and 20,507,026 between CAST and DBA/2J (Keane et al., 2011). From the two SNP annotation files, we only used SNPs where the C57BL/6J allele was shared between DBA/2J (shared SNP nr: $17,967,587)$. Finally, we used only exonic SNPs, resulting in a final number of 
1,513,184 SNPs. The Allelome.PRO "minread" parameter was set to 1 to include SNPs covered by one read. Genes with less than 10 read overlapping SNPs have been assigned to non-informative.

\section{Whole-genome bisulfite sequencing (WGBS) analysis}

The E6.5 ExE $\triangle$ G9a-Glp sample was isolated and processed into WGBS libraries using the Accel-NGS Methyl-seq kit as described in (Grosswendt et al., 2020). We utilized our previously generated ExE WT $(n=2), \Delta G 9 a(n=1)$ samples (Grosswendt et al., 2020) together with the generated $\triangle G 9 a-G L P$ sample to define G9a specific differentially methylated regions (DMRs) in E6.5 ExE. CpGs with less than ten reads were removed for the downstream analysis. CpGs were binned over a $1 \mathrm{~kb}$ window, filtering out all windows with less than $10 \mathrm{CpG}$. Window methylation levels were combined using the average. Next, we averaged WT and G9a knockout ( $\triangle G 9 a$ and $\triangle$ and GLP) methylation levels and calculated the delta (WT-KO). $\Delta$ me ExE DMRs were defined by havening a minimum difference of 0.2 to WT (|delta cutoff $\mid \geq 20 \%$ ). Feature enrichment of the identified DMRs over background was calculated for intergenic DMRs, genic ( $\pm 1 \mathrm{~kb}$ of TSS, RefSeq annotation), and different repeat classes (RepeatMasker tracks, UCSC) using the Fisher's exact test.

\section{ACKNOWLEDGEMENTS}

RNA sequencing and library preparation were performed at the Bauer Core Facility at Harvard University. AM was supported by NIH grants (DP3K111898 and P01GM099117) and the Max Planck Society.

\section{DATA AND CODE AVAILABILITY}

All datasets have been deposited in the Gene Expression Omnibus (GEO) and are accessible under GSE171206. Previously published data used in this study include WGBS data from GSE137337 for E6.5 ExE WT, $\Delta$ Dnmt1, $\Delta R n f 2, \Delta$ Eed, and $\Delta G 9 a$. 